\title{
Modeling Retest Effects in Developmental Processes Using Latent Change Score Models
}

\author{
Rohit Batra ${ }^{1}$, Silvia A. Bunge ${ }^{2}$, and Emilio Ferrer ${ }^{1}$ \\ ${ }^{1}$ University of California, Davis \\ ${ }^{2}$ University of California, Berkeley \\ Affiliation
}

\begin{abstract}
Author Note
Please note that this paper has been accepted for publication in Structural Equation Modeling Journal. For the peer-reviewed version of this paper, please visit: https://doi.org/10.1080/10705511.2021.1946807 Rohit Batra (D) https://orcid.org/0000-0001-8835-8573 Correspondence concerning this article should be addressed to Rohit Batra, University of California, Davis. E-mail: rbat@ucdavis.edu
\end{abstract}




\begin{abstract}
Studying development processes, as they unfold over time, involves collecting repeated measures from individuals and modeling the changes over time. One methodological challenge in this type of longitudinal data is separating retest effects, due to the repeated assessments, from developmental processes such as maturation or age. In this article, we describe several specifications of latent change score models using age as the underlying time metric and include parameters to account for retest effects. We illustrate the models with data on fluid reasoning collected from children and adolescents in a cohort-sequential design ranging from 6 to 20 years. Our models include alternative approaches to specify retest effects at the structural or measurement level of the model, and as an observed or a latent covariate. We discuss the benefits and limitations of the different approaches for univariate and multivariate data in the context of studying developmental processes.

Keywords: Latent Change Score Models, Retest Effects, Developmental Processes, Cohort Sequential Design
\end{abstract}




\section{Modeling Retest Effects in Developmental Processes Using Latent Change Score Models}

One important goal in developmental research is understanding the course of developmental processes, as they unfold over time. The concept of development cannot be conceived without the concept of time and, hence, longitudinal studies have become essential to the examination of developmental processes in individuals. Owing to the research in the past few decades, it has become clear that such an understanding requires information about how individuals change over time. For this, repeated assessments are needed. However, collecting repeated measures for the same individual brings its own methodological issues, since every new measure can be a function of the ability itself, maturation at the time of measurement (usually coded as age), or retest effects due to the repeated assessment. Estimating the true rate of change in an individual's development requires a formal partition of these important factors. The aim of this paper is to serve as a guide for researchers on how to account for retest effects in models of developmental change, particularly latent change score models.

\section{Retest Effects in Longitudinal Studies}

Longitudinal designs are necessary for the identification of intra-individual change (Baltes \& Nesselroade, 1979; Schaie \& Hertzog, 1983), inter-individual differences in intra-individual change and the different factors influencing developmental changes (Schaie

\& Hofer, 2001; Baltes \& Nesselroade, 1979). But with repeated assessments per individual, improvement due to practice (or other retest effects) can confound the true rate of change in the given trait (McArdle and Woodcock, 1997). Practice can be defined as the general familiarity with the testing environment and procedures (Rabbitt et al., 2004) or as a special retention ability of the task when done again and again which is unrelated to the ability or construct being measured. Throughout this paper, we will use the words practice and retest interchangeably.

Using latent growth curve models, McArdle and Woodcock (1997) discussed ways to 
separate the different components that can affect the changes in a variable over time, pointing out that the observed means and variances at every new occasion can be a function of age maturation as well as gains in practice from taking the test again. A few years later, Rabbitt et al. (2004) used a mixed-effects model with various cognitive tests and showed the importance of accounting for practice and the interactions between practice, individual differences in ability and task for estimating age-related changes accurately. Around the same time, Ferrer et al. (2004) and Ferrer et al. (2005), used multivariate mixed-effects models to examine longitudinal changes in memory, spatial capacity and speed ability. Some findings from these analyses include highest practice effects for memory and lowest for speed, indicating that the decline in memory can be masked by gains in practice. The authors highlighted the need to use age and retest together to explain changes (and correlates of changes) from occasion to occasion.

Moreover, age and retest are not the only confounds that can influence changes over time. Hoffman et al. (2011) examined via a simulation study the presence of age-related, retest and age-cohort effects (effects due to the age at which the subject joined the study). They found estimates of retest gains even when the generating models were specified to have none, and cautioned that what one may perceive as practice from a model might just be age-cohort or age-related effects. In all of this past work, one aspect is evident: the importance to separate the confounds due to age, retest or cohorts in models of change. But which model of change?

This paper focuses on modeling retest in latent change score (LCS) models (McArdle, 2009). LCS models were developed explicitly to model data that follow exponential forms, and have been extensively used in the analyses of developmental data, especially cognitive abilities (Estrada et al., 2020 Estrada \& Ferrer, 2019, Ferrer et al., 2007; Ferrer et al., 2010; Ghisletta \& Lindenberger, 2003, 2005; Grimm, 2008; Kievit et al., 2018; among others). LCS models offer a general framework, of which growth models such as a latent growth curve can be a special case. Although the previous approaches to study 
retest effects, such as mixed-effects models or growth curves, could be used, here we choose LCS models based on their following advantages: a) LCS are implemented within an SEM framework. This allows great flexibility for the specification of multiple model and parameter constraints; b) LCS models focus on the changes directly, and include dynamic parameters that capture influences of the previous states of the system on such changes (e.g., self-feedback and coupling parameters); c) LCS can include a measurement structure with manifest and latent variables, so it is possible to model change in the latent variables, as well as retest effects on either the latent or the manifest variables. Put simply, these models specify latent variables at each discrete time metric causing the manifest variables and the emphasis is on the changes within these latent variables. McArdle (2009) provides a comprehensive review of the different change modeling frameworks, for many of which the LCS model is a generalization. In LCS models, a latent change variable is defined as:

$$
\Delta y_{t}=\beta \cdot y_{t-1}+\cdot \alpha y_{s}
$$

where change in a latent variable $y$ at time $\mathrm{t}$ is a function of the previous time point latent variable $y_{t-1}$ with the coefficient of $\beta$, known as the auto-proportion parameter, and; the additive slope component $y_{s}$ which is a constant added to the changes at every time point with the coefficient of $\alpha$ (usually set to 1 for identification). These two components give this model the name of dual LCS model. We can also include a disturbance term at every time point in the definition which represents the dynamic variance. Previous work on retest used measurement occasions as the time metric for modeling growth for each individual (McArdle \& Woodcock, 1997; Rabbitt et al., 2004; Ferrer et al., 2004, 2005; Hoffman et al., 2011). In this report, we use discrete age bins to model changes in the ability over a given age span. In the next section, we discuss different choices of time that can be considered for specifying a LCS model, highlighting the advantages of using age as the underlying time metric for separating age from retest effects. 


\section{Coding Time using Discrete Age Bins}

The time metric underlying a given process plays a vital role in the understanding of a longitudinal model (Grimm \& Jacobucci, 2018; Hoffman et al., 2011; Miller \& Ferrer, 2017). Commonly used metrics when modeling developmental processes are measurement occasions, age or the grade level in school during the study. In LCS models, the time metric plays an equally important role for the specification and interpretation of the model. In models based on measurement occasions, the latent variables are specified for the first, second or $n^{\text {th }}$ occasion of measurement.

Age, on the other hand, is a continuous variable and since LCS models are usually conceptualized based on difference equations (for instance, $\Delta y_{t}=y_{t}-y_{t-1}$ ), their specification requires discrete age as the defining metric. One possible way to deal with this is where raw age is discretized into, say, full years (or other discrete age measure) called age bins, with as many bins as needed to cover the entire age span of the study. This procedure is commonly used in cohort-sequential designs where each individual has few assessments but, across all individuals, the data covers the entire age span (Ferrer, 2018). In more recent developments of LCS models, age can also be treated as continuous where change is defined instantaneously using differential equations (Voelkle and Oud, 2015).

In an occasion-based LCS model, the time points of measurement are used to specify the latent changes as differences between two consecutive occasions. But since every new latent change already has an additive slope component, it becomes impossible to differentiate the contribution for a new retest occasion from the additive slope, as both represent within-person changes across occasions. However, such a separation of within-person change from retest effects is possible in age-based models when there is enough variability in the retest interval (Ferrer et al., 2004), that is individuals differ from each other in their retest occasion during data collection for us to capture the change due to it. It is not just important to account for within-person variability (the interval of time between multiple occasions of the same subject can be different) but also the 
between-person variance (the interval of time between measurements of different subjects

can vary) (McArdle \& Woodcock, 1997; Estrada et al., 2020). The dynamic parameters of $\beta$ and $y_{s}$ (Equation 1) only account for within-person variability and occasion-based models fall short in accounting for between-person differences due to the interval of retest. However, we can overcome these issues inherent in occasion-based LCS models by using LCS models with age bins. Here, age itself becomes the underlying time metric and the goal is to quantify the change in a developmental process as a function of age - each latent change variable is defined at age bins and is a function of previous age and an additive slope component. Since each individual only has a few observations in the entire span of study, retest can now be introduced at every new assessment and is not confounded with the additive slope component. Hence, this approach makes it easier to account for within and between person variability in the retest interval without also having to account for age as an exogenous variable in the model.

\section{Purpose of the Study}

The purpose of this paper is to examine how retest effects can be included in latent change score models in order to enhance our understanding of developmental change in longitudinal studies. In the following section we describe data from a study of fluid reasoning ability across childhood and adolescence. We will use these data to illustrate various univariate and multivariate LCS specifications. Then, we discuss different conceptualizations of retest, and how they can be implemented in a LCS model. First, we include retest as a fixed covariate at the structural level, where latent changes are a function of the previous age bin, the additive slope component and an additional retest component (which can be varied for different age groups). Second, retest is used as a latent covariate at the structural level, a framework that may offer more flexibility in accounting for inter-individual differences in retest gains. Third, we use retest effects in second-order LCS models, where retest is included as a covariate at the measurement level, with the 
possibility of accounting for different retest gains across the different measures. Results and discussion follow with suggestions on further use of these models.

\section{Methods}

\section{Data and Measures Description}

Fluid reasoning (FR) is a construct that represents the ability to use logic and solve problems in new or unique situations, presumably independent of previously acquired knowledge (Cattell, 1987). It emerges early in life and shapes the way children acquire knowledge to do complex spatial, numerical or conceptual tasks (Ferrer et al., 2009). In this article, we use data from the Neural Development of Reasoning Ability (NORA) study (Ferrer et al., 2009, Wendelken et al., 2011) a longitudinal research project designed to examine the development of FR from childhood to adolescence. Data were collected using a cohort sequential design involving three waves of measurement with 201, 122 and 70 participants at the first, second and third occasion, respectively. At each occasion, participants completed four measures of fluid reasoning, namely the Block Design and Matrix Reasoning subtests of the Wechsler Abbreviated Scale of Intelligence (WISC-R; Wechsler, 1981), and the Analysis Synthesis and Concept Formation subtests of the Woodcock-Johnson Tests of Achievement (WJ-R; Woodcock et al., 1990).

Block Design measures the ability to arrange a set of red-and-white blocks in such a way as to reproduce a 2-dimensional visual pattern shown on a set of cards. Matrix Reasoning measures the ability to select the geometric visual stimulus that accurately completes a series of stimuli that change along a particular dimension. Analysis Synthesis measures the ability to analyze the components of an incomplete logic puzzle and to determine and name the missing components. Participants are given instructions on how to perform an increasingly complex procedure and receive feedback on the correctness of their responses. Concept Formation measures the ability to identify and state the rules for concepts when shown illustrations of both instances and non-instances of the concept 
(McArdle et al., 2002; Wendelken et al., 2016).

In the current work, our aim is to model the development of these measures and ultimately, the development of fluid reasoning with age (used as bins) as the underlying metric. Figure 1 shows the data collected for the four measures of fluid reasoning where scores from each individual with multiple assessments is connected by a line, and a dot is used to denote an individual with only one assessment for that particular measure. These plots show the within and between-person differences in the development of these measures from the age of 6 years to 15 years. For instance, Block Design (Figure 1(b)) shows a significant between-person variability for individuals starting at 6 years of age, where most individuals increase in their scores at their second assessment (some even with a drastic change) but there are also a few who show a decrease in their scores. Other measures follow variants of these increase-decrease patterns, with most individuals following different patterns within each measure. Hence, when accounting for the changes in the scores and development of these changes using age bins and retest, it becomes important to account for both within-person changes and between-person differences.

Very often researchers do not have multiple measures available at the measurement level to examine the development of a variable. To illustrate different approaches for assessing retest effects when only one manifest variable is available, we use a single measure of fluid reasoning. To derive this measure, Ferrer and colleagues (Wendelken et al., 2017; Ferrer, 2018) extracted factor scores from a confirmatory factor model that included the four measures of fluid reasoning at each measurement occasion and strong invariance conditions across the three occasions. This model had equality constraints in the factor loadings and intercepts, an initial factor mean fixed to zero, and a freely estimated factor mean for the second and third occasions. Using the scores from this factor variable, we created 15 age bins at discrete age intervals to represent the age-related changes from childhood to adolescence. Here, we use this single measure for illustrative purposes and acknowledge the limitations of estimated factor scores (Skrondal and Laake, 2001). Figure 
1(e) shows the plot of the composite fluid reasoning measure. Similar to the four observed measures, a trajectory of overall exponential increase can be seen in the scores of the fluid reasoning, and a need to account for within and between person differences in the study of changes.

The data in the current paper come from a cohort-sequential design. Participants were assessed on up to three occasions, with a retest interval ranging from 12 to 24 months across individuals. Approximately $60 \%$ of participants returned for a second assessment and about $35 \%$ for a third assessment. This design allows us to assume that what we measure as retest is some form of practice or familiarity with the testing conditions and not other sources of between-person differences (Ferrer, 2018).

\section{Specification of Retest Effects}

Including retest effects in a longitudinal model can be accomplished in different ways, each with potentially different implications. McArdle and Woodcock (1997) used the Memory and Reading tests from the Woodcock-Johnson-Revised scales to examine the presence of practice effects in reading and memory composite scores in a two-occasion retest design. They treated practice as another 'initial level' which starts at the second testing occasion $(t=1)$ and fit various growth curves with and without retest effects. Rabbitt et al. (2004) tested for the presence of practice effects in multiple cognitive tests, modeling variability between such tasks and variability among people of different age groups. Ferrer et al. (2004) expanded on the work by McArdle and Woodcock and used mixed models in which retest was allowed to be random across individuals in a variety of measures. In this paper, we build on this previous work and examine the changes in fluid reasoning across childhood and adolescence, with the goal of understanding such developmental changes as a function of age and retest. We propose different specifications of retest effects in the context of LCS models, specifying such effects at different levels of the model (structural and measurement) to help explain and separate the sources of 
variation due to age and retest.

\section{Retest effect as an observed covariate at the structural level}

In the first approach, we use a univariate LCS (1LCS, also called first-order LCS) and specify the retest effect as an exogenous covariate on the latent changes at the structural level. Here, the gain in practice is denoted by one-step increase in the value of the retest covariate. This specification allows one to determine how much of the latent change is influenced by this step gain in practice. By modeling retest at the structural level of a 1LCS where we use factor scores for modeling the trajectory of fluid reasoning, we are indicating that this practice represents a gain at the latent level of the ability being studied. Other work has focused on separating retest from other confounds such as cohort effects (Hoffman et al., 2011), which as mentioned before we do not assume exist for this data. However, group based differences can still exist in such data and we recommend a framework for that.

In a 1LCS, the true score latent variable can be written as:

$$
f r_{t, n}=f r_{t-1, n}+\Delta f r_{t, n}
$$

where, $f r_{t, n}(\mathrm{t}: 6, \ldots, 20)$ represents the true score at every new age bin for $n^{t h}$ individual and is specified as a sum of the true score at the previous age $(t-1), f r_{t-1}$; and the latent change for the current age $t$ indicated by $\Delta f r_{t}$. Comparing to Equation 1, we now have another component for retest in the definition of latent changes given as:

$$
\Delta f r_{t, n}=\beta \cdot f r_{t-1, n}+\alpha \cdot y_{s, n}+\gamma \cdot R_{t, n}
$$

Here, $R_{t, n}$ is the (n x 1) vector representing the step increase in practice gained at the current age, and $\gamma$ is the fixed coefficient that denotes the effect of retest on the latent changes. The two other components of the models, auto-proportion and additive slope, remain the same as in Equation 1. 
The value assigned to the step increase in retest can be specified in various ways, representing testable hypotheses (McArdle \& Woodcock, 1997; Ferrer et al., 2004). For instance, for $k$ number of measurements, retest basis coefficients can be fixed to be $R=(0,1,2, \ldots, k-1)$. Here, the retest function gets a one-unit step increase at every new measurement, hence, representing a linear effect of retest. Similarly, to explore nonlinear retest effects, the retest coefficients can be specified as latent basis (Ferrer et al., 2004). For example, for three-occasion data, the retest vector can be specified as $R=(0, \gamma, 1)$. This approach would be appropriate if, for instance, participants could show unequal retest effects across occasions, or when the researcher does not have a hypothesis about amounts of retest at the various assessment. If more measurement occasions were available for every individual, with more observations across age bins, then one could examine different nonlinear retest by estimating additional latent basis coefficients. Alternatively, one can also specify retest effects that remain constant after the second assessment, so each person can have a fixed basis retest vector of $R=(0,1,1)$. This approach can be especially useful in situations with few assessments per individual, as is the case in our data. We use this specification in our analysis.

A path diagram representing this model is included in Figure 2, This figure describes the observed variable for fluid reasoning $\mathrm{FR}_{t}$ measured at age bins of 6 to 20, where each manifest variable is caused by a latent factor representing fluid reasoning $f r_{t}$. The latent changes $\Delta f r_{t}$ from 7 to 20 years of age are a function of the true score at the previous age bin (with $\beta$ coefficient), the additive slope $y_{s}$, and the retest covariate $R_{t}$ (with $\gamma$ coefficient). Here, the paths of $\gamma$ are fixed but they can also be freely estimated as latent basis coefficients based on the researcher's hypotheses and the amount of information available for each individual from the data. Although not depicted here, this model can also include a dynamic variance that represents disturbances in the latent changes at every age.

An alternative model can be specified in which the covariate of retest is linked directly to the latent true score variable (instead of the latent changes). This model, 
however, is equivalent to the model described above and will give the same estimates as can be seen below:

$$
\begin{aligned}
f r_{t, n} & =f r_{t-1, n}+\Delta f r_{t, n} \\
& =f r_{t-1, n}+\beta \cdot f r_{t-1, n}+y_{s, n}+\gamma \cdot R_{t, n} \\
& =f r_{t-1, n}+\Delta f r_{t, n}+\gamma \cdot R_{t, n}
\end{aligned}
$$

In the next model, we examine the possibility of differences in retest effect across ages. For this, we fit a model where different age groups have different $\gamma$ coefficients. The specification for this model is given as:

$$
\Delta f r_{t, n}=\beta \cdot f r_{t-1, n}+y_{s, n}+\gamma_{i} \cdot R_{t, n}
$$

where all the above variables are the same as before but the $\gamma$ coefficient now has a subscript i that can take different values based on different age groups. In our example, we compare three age groups, where the coefficients are specified as: $\gamma_{1}$ for subjects from 6-10 years; $\gamma_{2}$ for subjects from 11-15 years, and; $\gamma_{3}$ for subjects from 16-20 years. Each $\gamma_{i}$ represents the proportional increase (or decrease) in the latent changes for every new assessment for an individual. This helps in assessing whether age-based differences exist within the data from a repeated assessment. A path diagram of this model is presented in Figure 3. Here, groups of age bins have different retest $\gamma_{i}$ coefficients based on their age. This could be a way to check for cohort effects along with a retest coefficient and the related group here acting as a cohort in the model. In our dataset, the number of observations available across age bins is small (20 observations per age bin, on average, and the $20^{\text {th }}$ age bin with the fewest observations from 9 individuals), which limits our ability to examine a large number of cohorts. 


\section{Retest effect as a latent variable at the structural level}

With the next approach, we specify retest as an exogenous latent variable. This approach offers the flexibility of estimating variance in the retest variable, which can account for the between-person differences in the amount of retest. Because retest is treated as latent, its loadings can now be specified based on one's hypothesis. For example, a linear practice effect can be tested with basis coefficients as $(0,1,2, \ldots)$. Alternatively, these coefficients can be specified as latent and estimated from the data, such as $(0, \gamma, 1)$ for a subject with three measurements. Using retest as a latent variable, the equation of changes in fluid reasoning $f r_{t, n}$ can be written as:

$$
\Delta f r_{t, n}=\beta \cdot f r_{t-1, n}+y_{s, n}+\gamma \cdot y_{p, n}
$$

where, as before, the changes are a function of the previous age bin $t$ with the auto-proportion coefficient, the additive latent slope component $y_{s, n}$, for which we estimate a mean $\mu_{s}$ and a variance $\sigma_{s}^{2}$, and a latent covariate of practice $y_{p, n}$, with a vector of fixed coefficients for the three occasions of measurement $\gamma=(0,1,1)$.

Now that we model practice as latent, this allows for the estimation of the latent practice mean $\mu_{p}$ and variance $\sigma_{p}^{2} \cdot \mu_{p}$ indicates the average gain in latent changes for every new measurement occasion and the variance $\sigma_{p}^{2}$ indicats the variability among individuals in the amount of gain from taking the test repeatedly. It is important to separate the effect of practice from the additive slope component at the structural level which we accomplish by modeling a specific hypothesis of the effect of the latent variable of practice on changes at every new assessment. The fixed retest basis coefficients test the hypothesis that retest happens at the first repeated assessment and remains constant at the the next assessment. This kind of specification can also be used when the data available at each occasion is

sparse. In our model, we do not covary the practice latent variable with the initial level, assuming that the amount of gain in retest is not related to fluid reasoning at the first measurement occasion. Figure 4 shows a path diagram for this model where practice is 
now represented by a circle as an exogenous latent variable influencing the latent changes.

\section{Retest effect as an observed covariate at the measurement level}

Till now, our discussion has been with respect to univariate LCS models, where a single manifest variable (often, a composite score of multiple indicators) is used with the assumption that it represents the same construct at each measurement occasion, a property called measurement invariance (Meredith, 1993). Establishing longitudinal measurement invariance is important in latent variable modeling to ensure the measurement tool is consistently measuring the same construct across repeated measures (Widaman et al., 2010). However, this assumption is not testable in univariate models, in which each occasion consists of one indicator only.

These limitations can be overcome with second-order latent change score models (2LCS), a multivariate extension of 1LCS models where, instead of a single manifest variable, there are multiple indicators loading onto the latent factor at each occasion. This modeling approach accounts for the measurement structure, making it possible to test if the same construct is being measured across occasions and also separate the variance due to error from the observed measures (Ferrer et al., 2008). In our specification, we use strict factorial invariance, with factor loadings, intercepts, and unique variances of each observed indicator constrained to be equal across all age bins. We also estimated the covariances of the residuals of the same indicators at lag 1 (eg., $\left.\operatorname{Cov}\left(m r_{t}, m r_{t+1}\right)\right)$. Scaling was done by fixing the loading of Matrix Reasoning to 1 and the mean of the latent initial level to 0 (Ferrer et al., 2008).

With multiple indicators at the measurement level of the LCS model, an interesting question can now be answered: "Is the gain in practice the same across all manifest variables?" For instance, if a participant completed tests of matrix reasoning and block design at two occasions during the study, was the gain in practice the same or different for matrix reasoning and block design? Another interesting question is: "With multiple 
occasions of measurement per participant, what if the gain in practice is different for different occasions for each of the observed indicators?" For instance, if we observe difference in retest coefficients for the four measures, is it possible that each measure differs in the retest basis vector they use for the overall data. To answer these questions, we first write the generating model for our proposed 2LCS with an additive component for retest at the measurement level, as:

Measurement Model:

$$
\begin{aligned}
\mathrm{MR}_{t, n} & =\tau_{m r}+\lambda_{m r} \cdot f r_{t, n}+\gamma \cdot R_{t, n}+e_{m r(t, n)} \\
\mathrm{BD}_{t, n} & =\tau_{b d}+\lambda_{b d} \cdot f r_{t, n}+\gamma \cdot R_{t, n}+e_{b d(t, n)} \\
\mathrm{CF}_{t, n} & =\tau_{c f}+\lambda_{c f} \cdot f r_{t, n}+\gamma \cdot R_{t, n}+e_{c f(t, n)} \\
\mathrm{AS}_{t, n} & =\tau_{a s}+\lambda_{a s} \cdot f r_{t, n}+\gamma \cdot R_{t, n}+e_{a s(t, n)}
\end{aligned}
$$

Structural Model:

$$
\begin{aligned}
\Delta f r_{t, n} & =\alpha \cdot y_{s, n}+\beta \cdot f r_{t-1, n}+v_{\Delta(t, n)} \\
f r_{t, n} & =f r_{t-1, n}+\Delta f r_{t, n}
\end{aligned}
$$

Here, MR, BD, CF, and AS represent the four observed measures of fluid reasoning, namely, Matrix Reasoning, Block Design, Concept Formation, and Analysis Synthesis. For each variable, the measurement model includes several components: $\tau$ is the indicator specific intercept constrained to be equal across all age bins; $\lambda$ is the factor loading with which the indicator loads onto the true score factor $f r_{t, n}$ at age bin $t$, also constrained to be equal across age; $\gamma$ is the retest coefficient for the retest variable $R_{t, n}$, which is a vector of $(0,1,1)$ for (at most) three occasions for each $n^{\text {th }}$ individual.

As illustrated in Figure 1, the trajectory for each measures is not just a step gain across all individuals. One alternative way to capture this variation is to specify every individual with their respective pattern of improvement. For example, for an individual with scores of Block Design of $(50,59,68)$, linear retests can be specified as $(0,1,2)$, whereas for another individual with scores of $(51,61,59)$, retest can be specified as an 
up-down pattern $(0,1,0)$. However, such an individualized assignment of retest can be detrimental to the interpretation of the $\gamma$ coefficient since it represents the effects on changes at the population level. When enough data (with retest variation) is available for each individual, then this technique can be used to estimate $\gamma$ for each individual. Hence, for the current analysis of fluid reasoning, we use one-step gain retest effect. In the structural model, the specification of latent true score and latent changes remains the same as before but we now add dynamic errors denoted by $v_{\Delta(t, n)}$, allowing individuals to vary in their latent change trajectories.

We start with a model in which retest coefficient is fixed to be equal across all indicators, assuming the gain in practice is equivalent across all manifest variables. Next, we relax this constraint and specify the measurement model such that the $\gamma$ coefficients are different, allowing each manifest variable to have different retest effects. The generating (measurement) model can be specified as the following, while the structural model remains the same as above:

$$
\begin{aligned}
\mathrm{MR}_{t, n} & =\tau_{m r}+\lambda_{m r} \cdot f r_{t, n}+\gamma_{m r} \cdot R_{t, n}+e_{m r(t, n)} \\
\mathrm{BD}_{t, n} & =\tau_{b d}+\lambda_{b d} \cdot f r_{t, n}+\gamma_{b d} \cdot R_{t, n}+e_{b d(t, n)} \\
\mathrm{CF}_{t, n} & =\tau_{c f}+\lambda_{c f} \cdot f r_{t, n}+\gamma_{c f} \cdot R_{t, n}+e_{c f(t, n)} \\
\mathrm{AS}_{t, n} & =\tau_{a s}+\lambda_{a s} \cdot f r_{t, n}+\gamma_{a s} \cdot R_{t, n}+e_{a s(t, n)}
\end{aligned}
$$

Here, for instance, $\gamma_{m r}$ represents the effects of a repeated assessment on matrix reasoning, which might be different from say, $\gamma_{b d}$, denoting the retest coefficient of block design. Figure 5 gives a path diagram for this model. In this figure, the variable retest has arrows leading to the observed indicators, with different $\gamma$ coefficients denoting the possibility of distinct effects. The first bin of $f r_{6}$ does not have arrows since there is no retest at the first time in the study period. This model also includes residual covariances at lag 1 given by the two-headed arrows between two consecutive indicators while the factor loadings and unique variances are shown to be invariant across bins. We also estimate a 
residual dynamic variance denoted by $\sigma_{\phi}^{2}$ which measures if inter-individual variability exists in the latent changes. We also estimate the intercepts of the manifest variables which have not been depicted in the figure for the sake of simplicity.

\section{Results}

In this section, we cover the results of the three specifications for modeling retest. We used different softwares and packages, depending on our modeling needs and software capabilities. The code used for all the analyses can be found at [URL masked for blind review]. Here, our aim is to highlight the results from each alternative approach but the choice of the approach by a researcher depends on a number of factors and methodological considerations, which we describe in the discussion section. Accordingly, when comparing among models in our analyses, we use fit indices (e.g., AIC and BIC for non-nested models) together with other criteria, and do not recommend using fit statistics as the sole criteria for model comparison.

\section{Univariate LCS Model with Retest as an Observed Covariate}

In the first set of analyses, we used retest as an observed covariate at the structural level. In our data (which follows a cohort sequential design), each participant may have one, two, or three observations across the 15 discrete age bins. This incomplete data design was constructed with the aim of obtaining approximately the same number of observations across all ages (Ferrer, 2018). For the following analyses, we assume the data are missing at random. All analyses in this section were carried out using the lavaan package in $\mathrm{R}$ (Rosseel, 2012).

We first fit a LCS model that does not include any retest effects which will serve as a baseline for comparison. This model had an auto-proportion parameter significantly different from zero $(\beta=-.193 ; p<.05)$. That is, the latent change at any age bin $t$ depends on the latent variable fluid reasoning at the previous age bin $(t-1)$, with a proportion of -.193. This implies that the changes in fluid reasoning are being slowed down 
more and more as we move forward in the developmental age. The changes are also comprised of an additive slope $\left(\mu_{s}=.209 ; p<.05\right)$ that represents average gains of .21 units for every year. In total, this model includes seven parameters (all reliably different from zero, $p<.05)$ and the RMSEA fit index was .021 indicating a good model fit. The results are included in the second column of Table 2

Next, we fit a LCS model with retest as a predictor of the latent variables representing fluid reasoning. As described in the previous sections, this model was equivalent to a model where retest predicts the latent changes. Compared to the previous model without retest, comparative fit indices like AIC and BIC show an improvement in fit. The retest coefficient was significantly different from zero $(\gamma=.199 ; p<.05)$, indicating that at their second assessment, individuals' fluid reasoning scores increased by .199 units. The mean of the additive slope shifted down $\left(\mu_{s}=.154 ; p<.05\right)$ but the variance remained almost the same $\left(\sigma_{s}^{2}=.008 ; p<.05\right)$. This small additive slope variance indicates that individuals do not vary substantially in their constant shift in changes from one age bin to the next. The rest of the parameters in the model gave similar estimates to those from a model without retest effects (with all significant different from zero; $p<.05$ ). These results are included in the third column of Table 2 .

Figure 6 includes a comparison of three individuals and their estimated trajectories when retest is included in the model as a predictor of the latent scores. The black line represents the estimated trajectory of fluid reasoning from a model without retest. As can be seen, this line is not an appropriate estimate for the three individuals, whose raw scores are indicated as dots (each with a respective color). For instance, ID 1's raw scores, depicted in orange, were measured at ages 12,14 and 16. When retest was included as part of the expected trajectory for this individual, we notice a stepwise increase at the second assessment (age 14) and another increase at the third assessment (age 16). Before the first observation, this person's expected values are the same as those denoted by the black line since there is no retest until that point, so the population and the individual model is the 
same. We also notice that, once this individual receives all retests, the estimated trajectory goes down, which is expected from this model where rate of decline is given by the $\beta=-.203$. Similarly, the second individual (ID 10) has observations at ages 9,11 and 12, with an expected trajectory depicted by the green line; the third individual (ID 143) has observation at ages 18 and 19, with an expected trajectory represented by the blue line.

To examine differences in the retest gains across age, we next fit a model that included different coefficients for three age groups: ages 6-10, 11-15, and 16-20, represented by $\gamma_{1}, \gamma_{2}$, and $\gamma_{3}$, respectively. The results from this model are included in the last column of Table 2, The estimates of the $\gamma_{i}$ coefficients are all significantly different from zero $\left(\gamma_{1}=.184, \gamma_{2}=.227, \gamma_{3}=.175 ; p<.05\right)$ and show that practice-related gains are different for different age groups. For example, for children 6-10 years-old and adolescents 16-20 years-old, the estimated retest effect was .18, whereas for those in 11-15 age group, it was .23 , indicating a higher change due to the repeated assessments for this age group. As indicated by AIC and BIC, this model also gave a better fit than the first model without any retest effect. When using comparative fit indices, however, this model did not fit better than a model with only one coefficient of retest. Nonetheless, if a researcher has a hypothesis of cohort differences, we would recommend this approach, which indicates evidence for group differences in retest gains across age bins.

\section{Univariate LCS Model with Retest as a Latent Covariate}

Next, we fit retest as a latent variable with the purpose of examining individual differences in retest gains among individuals. For this, we used dynr package (Ou et al., 2017), which allows estimating a variance component for retest across subjects in the study.

As compared with the model where retest is treated as a predictor, described in the previous section, we notice quite similar estimates, with an auto-proportion parameter of $\beta=-.201(p<.05)$, reflecting a similar reliance of latent changes on latent fluid reasoning the previous year, but now with an added effect of retest. The retest mean 
$\left(\mu_{p}=.199 ; p<.05\right)$ is now the same as the estimated coefficient from the previous model $(\gamma=.199 ; p<.05)$, indicating the average gains in changes due to retest whenever individuals in the study have a new measurement. These two have the same values because when we use retest as a latent predictor, the estimation essentially flips and the average gains indicated by $\gamma$ previously are now indicated through the mean of the retest latent variable. However, the variance of retest was not significantly different from zero $\left(\sigma_{p}^{2} \approx 0 ; p>.05\right)$, indicating a lack of variability in retest across individuals. Since the number of individuals available at the second and third assessment is smaller (122 and 70 participants respectively), it is possible that this model could not detect variance in retest reliably. Compared to the model without any retest in Table 2, AIC and BIC showed an improvement in model fit. The model included a total of eight parameters, and their estimates are provided in Table 3.

\section{Multivariate LCS Model with Retest as a Covariate at the Measurement Level}

In the next set of analysis, we implement a multivariate LCS model (2LCS) and include retest as a covariate at the measurement level. As described previously, we imposed strict invariance and allowed for the residual covariances at lag 1 to be estimated for each indicator. Since most individuals have observations at multiple indicators when they came back for their second or third assessments and the measures are supposed to capture the same construct, the retest predictors for the different measures are expected to show high correlations. This could cause convergence issues if appropriate starting values are not fed into the program. All these models were fitted using Mplus modeling software (Muthén and Muthén, 2019).

The first model we examined was a 2LCS without retest effects, and this served as a baseline model for comparison. The change parameters in this model included an auto-proportion $(\beta=-.193 ; p<.05)$ representing feedback from the previous age, and an additive component $\left(\mu_{s}=3.85 ; p<.05\right)$, denoting increments at every age bin. However, 
the variance of the additive slope was not significant $\left(\sigma_{s}^{2}=.165 ; p>.05\right)$, implying no perceptible differences in this additive component across individuals. The full results from this model are reported in the first column of Table 1 .

The next model examined the hypothesis of equal retest effects across all manifest variables (i.e., all $\gamma$ coefficient equal across the indicators). Relative to the baseline model, this model yielded better fit based on the AIC and BIC fit comparison criteria. The parameter estimates included an auto-proportion parameter with an increase value $(\beta=-.181 ; p<.05)$, indicating a higher rate of slowing down as we move along age in the study. Importantly, the model showed a retest effect of 1.57 units $(\gamma=1.572 ; p<.05)$ for the repeated assessment, for each of the indicators. Relative to the univariate model in Table 2, where retest is a predictor of the latent scores, the retest coefficient is quite different. This might be because the four indicators used in the measurement part have different metrics. Thus, the step gain of 1.57 units might mean something different for each of the manifest variables. The mean of the additive slope component also decreased from the previous model (from 3.85 to 3.28 ), indicating that the constancy of changes is decreased when we include retest in the model. The variance of the additive slope, however, remained not different from zero. In total, this model included 22 parameters, and their estimates are reported in the third column of Table 1 .

Our next hypotheses evaluated whether the retest effects differed for each indicator of the latent fluid reasoning factor. This model is represented by Equation 10 and Figure 5 . Each indicator in the model is now a function of the fluid reasoning latent true score, with a unique factor loading and a unique retest effect whenever there is a repeated assessment. In total, this model included 25 free parameters and the model fit was quite similar to the previous model based on the AIC and BIC fit criteria. The auto-proportion coefficient is larger than in the previous model $(\beta=-.178 ; p<.05)$, reflecting a lower rate of slowing down in fluid reasoning than before. Out of the four retest estimates $\gamma$, only that for Block Design is not significantly different from zero. There is a noticeable difference in the retest 
coefficient for the other three indicators, with estimates of 2.23 for Matrix Reasoning, 2.23 for Concept Formation, and .97 for Analysis Synthesis. The mean of the additive slope is significantly different from zero $\left(\mu_{s}=3.15 ; p<.05\right)$, but its variance is not $\left(\sigma_{s}^{2}=.104 ; p>.05\right)$, reflecting that the constant changes in fluid reasoning for every new age bin are not different across individuals. We also examined the expected latent trajectories of fluid reasoning when retest is included at the structural level of a multivariate LCS (same as the first approach but now with multiple indicators) and at the measurement level, with both models yielding similar trajectories (Figure included in Appendix).

\section{Discussion}

\section{Summary of Findings}

With this paper, we examined several possible specifications for modeling retest in univariate and multivariate LCS models. The aim of this approach was to examine the effect of repeated measurements and separate it from other possible factors affecting developmental changes, in our case, defined by age. We used discrete age bins to define the progression of fluid reasoning and separate the variance due to age from that due to retest. Our goal with the current work is to outline steps needed to estimate a model that allows partitioning of the variance between age and retest based on some of these criteria: 1) data design; 2) time metric; 3) number of measures available; 4) number of assessments available; 5) assumption of retest occurrence (e.g., one-step or linear).

We used four measures designed to represent fluid reasoning, and implemented a LCS model to capture its exponential form from childhood to adolescence (McArdle et al., 2002). Examining possible mechanisms underlying the development of fluid reasoning across age can become a challenging task and we discuss the findings from each of the alternative ways we can modify the LCS model to account for retest effects. In our first proposed approach, we specify retest as an observed predictor of latent changes in a 
univariate LCS. In a LCS model, the $\beta$ parameter shows how the influence of previous observations on the changes accumulates over age, whereas the additive slope reflects constancy in the changes across ages. Here, the parameter $\beta$ decreases when retest is included in the model, reflecting that the rate of slowing down of the changes is underestimated when the model does not account for gains through retest. This can be seen in Figure 7 where we compared the expected changes in fluid reasoning from a model with and without retest effects.

The next model specification defined retest as a latent variable, and the aim of this model was to identify possible differences in retest effects among individuals. The results of this model, however, indicate that the variance for retest is not significantly different from zero. This result is not surprising since most individuals do not have long intervals between their retest measurements (at most 2 years between any two consecutive assessments) and the one-step gain assumption for the coefficient of latent practice variable may be suitable for these data. In spite of this lack of statistical significance, this approach can be helpful in finding evidence of inter-individual variability in the gains of retest in other datsets. The parameter estimates from this model were similar to those from a model in which retest was specified as an observed predictor of the latent changes.

In the next step, we extended these univariate models to a multivariate LCS specification in which retest was included at the measurement level. A key goal in this approach was to evaluate if a single retest coefficient was sufficient to represent retest gains for the entire multivariate system or if the different measures showed specific retest gains. When there is missingness across measures for an individual; that is, not all measures were completed at any given assessment by an individual - this makes it impossible to be able to include retest at one particular discrete assessment at the structural level. Hence, including retest at the measurement level can be advantageous in such situations. Our results indicate that a model with different coefficients for the different indicators was more representative of retest gains. This is very reasonable given that not all the manifest 
variables use the same scale and, thus, assigning a single retest coefficient might underestimate the retest produced by the model. However, we see that this doesn't work for all measures. We refer the reader to the plot of Block Design in Figure 1 which shows significant variability in the trajectories across individuals: some increase and stay around the same score; some increase and then decrease in their scores while some decrease in their scores. Hence, a model that assigns a one-step increase in retest to all individuals might be too restrictive. This might explain the fact that Block Design was the only variable whose retest coefficient was not significantly different from zero among all the retest predictors at the measurement level. If individuals differ in their pattern of retest for a single measure, then, a more individualized approach is needed to account for different gains (or losses) in retest.

\section{Methodological Considerations}

Existing work recommends taking caution in the study design, as within-person changes can masquerade as retest effects (Hoffman et al., 2011). In this report we outline different ways to account for retest in a design where other confounds are assumed to be either negligible or parameterized differently. The model specifications proposed here can be used to check for other within-person changes that can be coded as step changes for every new measurement at the level of an individual. For instance, fatigue effect can be represented with similar mechanisms, which works as the opposite of retest effect when a researcher has large number of testing occasions for individuals (daily diary studies) and can lead to overestimates in latent developmental trajectories.

In a LCS model with discrete time, the latent scores are a linear function of the latent scores at the previous time and the latent changes between the two time points. Re-expressing Equation 1 in terms of fluid reasoning without any changes, gives the 
expression:

$$
f r_{t}=(1+\beta) \cdot f r_{t-1}+y_{s}
$$

where each latent score is a function of the previous latent score with the effect of (1 $+\beta)$ and a constant. This effect due to the coefficient gets compounded for every new latent score and, as the process moves forward across age, the rate of how much the score relies on the initial state of the process is determined by the auto-proportion parameter $\beta$ (see, Hamagami \& McArdle, 2019 for a detailed explanation). Hence, negative values of $\beta$ denote a deceleration rate, that is the changes are damped as we progress forward in age. When modeling retest at the measurement level in a multivariate LCS model, we noticed that this rate of deceleration was slower once we accounted for retest as a predictor of the measures, relative to when retest was included at the latent level in a univariate model. This might be because we are first separating the observed measures from their retest contribution, and then separating the true score variance, which might affect the value of true rate of changes in developmental improvements in fluid reasoning. This structure is also affected by not having direct auto-regressive effect among the indicators: that is, the indicator of Matrix Reasoning at the $k^{\text {th }}$ age bin does not influence the indicator of Matrix Reasoning at the $(k+1)^{\text {th }}$ age bin, unlike the true score fluid reasoning latent variables. Hence, once an individual receives a retest effect at the measurement level, it does not propagate to subsequent occasions.

Our work in this paper treats time as a discrete entity with the use of age bins, in what could be referred to as a pseudo-continuous framework (Ferrer, 2018). Recently, there has been considerable work on continuous time modeling in structural equation and state space modeling, where the dynamics of repeated measures are modeled using a single underlying continuous generating process (Voelkle et al., 2012; Ji \& Chow, 2019). Here, our focus is limited to discrete age, but one possible extension is implementing these proposed models in continuous time, where the $\gamma$ coefficient would represent an instantaneous retest 
effect for every new measurement. However, since a continuous model is assumed to underlie any infinitesimally small interval, including retest effects using step gains might not be simple, as such effects are discrete and, thus, we do not know at which exact instant can the effect of retest be accounted for in such a process.

Another possible extension of our work is including models that accommodate measures that vary throughout the study period. It is often the case that measures in developmental studies change across time due to, say, age-appropriate assessments, measures become obsolete, etc. (McArdle, 2009). However, even with a design where invariance across time points is established by overlapping variables over measurement occasions (McArdle et al., 2009), retest can still influence true scores and changes in the ability if the same measure is being used at least twice. Separating retest in such a framework where the measures change can be an interesting extension of the models proposed in this paper.

Identifying retest effects in longitudinal studies is not always straightforward. If the aim of a study is to understand the mechanisms that lead to developmental changes, there is a need to account for retest to attain a clear understanding of the said developmental process. We hope this paper is a step in that direction and motivates other researchers to consider different possibilities for separating retest effects from other developmental confounds. 


\section{References}

Baltes, P. B., \& Nesselroade, J. R. (1979). History and rationale of longitudinal research. Longitudinal research in the study of behavior and development (pp. 1-39). Academic Press.

Cattell, R. B. (1987). Intelligence: Its structure, growth and action. Elsevier.

Estrada, E., \& Ferrer, E. (2019). Studying developmental processes in accelerated cohort-sequential designs with discrete-and continuous-time latent change score models. Psychological methods, 24(6), 708.

Estrada, E., Hamagami, F., \& Ferrer, E. (2020). Estimating age-based developmental trajectories using latent change score models based on measurement occasion. Multivariate behavioral research, 55(3), 454-477.

Ferrer, E. (2018). Discrete-and semi-continuous time latent change score models of fluid reasoning development from childhood to adolescence. Longitudinal Multivariate Psychology. Routledge.

Ferrer, E., Balluerka, N., \& Widaman, K. F. (2008). Factorial invariance and the specification of second-order latent growth models. Methodology, 4(1), 22-36.

Ferrer, E., McArdle, J. J., Shaywitz, B. A., Holahan, J. M., Marchione, K., \& Shaywitz, S. E. (2007). Longitudinal models of developmental dynamics between reading and cognition from childhood to adolescence. Developmental psychology, $43(6), 1460$.

Ferrer, E., O’Hare, E. D., \& Bunge, S. A. (2009). Fluid reasoning and the developing brain. Frontiers in neuroscience, 3, 3.

Ferrer, E., Salthouse, T. A., McArdle, J. J., Stewart, W. F., \& Schwartz, B. S. (2005). Multivariate modeling of age and retest in longitudinal studies of cognitive abilities. Psychology and aging, 20(3), 412. 
Ferrer, E., Salthouse, T. A., Stewart, W. F., \& Schwartz, B. S. (2004). Modeling age and retest processes in longitudinal studies of cognitive abilities. Psychology and aging, $19(2), 243$.

Ferrer, E., Shaywitz, B. A., Holahan, J. M., Marchione, K., \& Shaywitz, S. E. (2010). Uncoupling of reading and iq over time: Empirical evidence for a definition of dyslexia. Psychological science, 21 (1), 93-101.

Ghisletta, P., \& Lindenberger, U. (2003). Age-based structural dynamics between perceptual speed and knowledge in the berlin aging study: Direct evidence for ability dedifferentiation in old age. Psychology and aging, 18(4), 696.

Grimm, K. J. (2008). Longitudinal associations between reading and mathematics achievement. Developmental neuropsychology, 33(3), 410-426.

Grimm, K. J., \& Jacobucci, R. (2018). Individually varying time metrics in latent change score models 1. Longitudinal Multivariate Psychology, 61-79.

Hamagami, F., \& McArdle, J. J. (2019). Latent change score models with curvilinear constant bases. Longitudinal multivariate psychology, 80-108.

Hoffman, L., Hofer, S. M., \& Sliwinski, M. J. (2011). On the confounds among retest gains and age-cohort differences in the estimation of within-person change in longitudinal studies: A simulation study. Psychology and aging, 26(4), 778.

Ji, L., \& Chow, S.-M. (2019). Methodological issues and extensions to the latent difference score framework. Longitudinal multivariate psychology, 9-37.

Kievit, R. A., Brandmaier, A. M., Ziegler, G., Van Harmelen, A.-L., de Mooij, S. M., Moutoussis, M., Goodyer, I. M., Bullmore, E., Jones, P. B., Fonagy, P., et al. (2018). Developmental cognitive neuroscience using latent change score models: A tutorial and applications. Developmental cognitive neuroscience, 33, 99-117.

McArdle, J. J. (2009). Latent variable modeling of differences and changes with longitudinal data. Annual review of psychology, 60, 577-605. 
McArdle, J. J., Ferrer-Caja, E., Hamagami, F., \& Woodcock, R. W. (2002). Comparative longitudinal structural analyses of the growth and decline of multiple intellectual abilities over the life span. Developmental psychology, 38(1), 115.

McArdle, J. J., Grimm, K. J., Hamagami, F., Bowles, R. P., \& Meredith, W. (2009). Modeling life-span growth curves of cognition using longitudinal data with multiple samples and changing scales of measurement. Psychological methods, 14 (2), 126.

McArdle, J. J., \& Woodcock, R. W. (1997). Expanding test-retest designs to include developmental time-lag components. Psychological Methods, 2(4), 403.

Meredith, W. (1993). Measurement invariance, factor analysis and factorial invariance. Psychometrika, 58(4), 525-543.

Miller, M. L., \& Ferrer, E. (2017). The effect of sampling-time variation on latent growth curve models. Structural Equation Modeling: A Multidisciplinary Journal, 24(6), $831-854$

Muthén, L., \& Muthén, B. (2019). Mplus. The comprehensive modelling program for applied researchers: user's guide, 5 .

Ou, L., Hunter, M. D., \& Chow, S.-M. (2017). What's for dynr: A package for linear and nonlinear dynamic modeling in r. Journal of Statistical Software.

Rabbitt, P., Diggle, P., Holland, F., \& McInnes, L. (2004). Practice and drop-out effects during a 17-year longitudinal study of cognitive aging. The Journals of Gerontology Series B: Psychological Sciences and Social Sciences, 59(2), P84-P97.

Rosseel, Y. (2012). Lavaan: An r package for structural equation modeling and more. version 0.5-12 (beta). Journal of statistical software, 48(2), 1-36.

Schaie, K. W., \& Hertzog, C. (1983). Fourteen-year cohort-sequential analyses of adult intellectual development. Developmental Psychology, $19(4), 531$.

Schaie, K. W., \& Hofer, S. M. (2001). Longitudinal studies in aging research.

Skrondal, A., \& Laake, P. (2001). Regression among factor scores. Psychometrika, 66 (4), $563-575$. 
Voelkle, M. C., \& Oud, J. H. (2015). Relating latent change score and continuous time models. Structural Equation Modeling: A Multidisciplinary Journal, 22(3), 366-381.

Voelkle, M. C., Oud, J. H., Davidov, E., \& Schmidt, P. (2012). An sem approach to continuous time modeling of panel data: Relating authoritarianism and anomia. Psychological methods, 17(2), 176.

Wechsler, D. (1981). Wais-r manual: Wechsler adult intelligence scale-revised. Psychological Corporation.

Wendelken, C., Ferrer, E., Ghetti, S., Bailey, S. K., Cutting, L., \& Bunge, S. A. (2017). Frontoparietal structural connectivity in childhood predicts development of functional connectivity and reasoning ability: A large-scale longitudinal investigation. Journal of Neuroscience, 37(35), 8549-8558.

Wendelken, C., Ferrer, E., Whitaker, K. J., \& Bunge, S. A. (2016). Fronto-parietal network reconfiguration supports the development of reasoning ability. Cerebral Cortex, $26(5), 2178-2190$.

Wendelken, C., O'Hare, E. D., Whitaker, K. J., Ferrer, E., \& Bunge, S. A. (2011). Increased functional selectivity over development in rostrolateral prefrontal cortex. Journal of Neuroscience, 31 (47), 17260-17268.

Widaman, K. F., Ferrer, E., \& Conger, R. D. (2010). Factorial invariance within longitudinal structural equation models: Measuring the same construct across time. Child development perspectives, 4(1), 10-18.

Woodcock, R. W., Johnson, M. B., \& Mather, N. (1990). Woodcock-johnson psycho-educational battery-revised. DLM Teaching Resources. 


\section{Table 1}

Parameters of Multivariate LCS with/without retest covariate (including residual covariances)

\begin{tabular}{|c|c|c|c|}
\hline Parameter & $2 \mathrm{LCS}$ & $\begin{array}{l}\text { 2LCS with } \\
\text { indicators } \sim \gamma\end{array}$ & $\begin{array}{l}\text { 2LCS with } \\
\text { indicators } \sim \gamma_{i}\end{array}$ \\
\hline \multicolumn{4}{|l|}{ Loadings: } \\
\hline$\lambda_{m r}$ & $1^{+}$ & $1^{+}$ & $1^{+}$ \\
\hline$\lambda_{b d}$ & $2.765(.140)$ & $2.881(.153)$ & $2.916(.163)$ \\
\hline$\lambda_{c f}$ & $1.215(.061)$ & $1.226(.064)$ & $1.234(.068)$ \\
\hline$\lambda_{a s}$ & $.750(.042)$ & $.727(.045)$ & $.761(.048)$ \\
\hline \multicolumn{4}{|l|}{ Regressions: } \\
\hline$\beta$ (auto-proportion) & $-.193(.022)$ & $-.181(.022)$ & $-.178(.022)$ \\
\hline$\gamma$ (retest coefficient) & - & $\gamma=1.572(.284)$ & $\begin{array}{l}\gamma_{m r}=2.226(.451) \\
\gamma_{b d}=2.104(1.123)^{n s} \\
\gamma_{c f}=2.232(.554) \\
\gamma_{a s}=.972(.362)\end{array}$ \\
\hline \multicolumn{4}{|l|}{ Factor Means: } \\
\hline$\mu_{0}$ & $0^{+}$ & $0^{+}$ & $0^{+}$ \\
\hline$\mu_{s}$ & $3.854(.384)$ & $3.276(.359)$ & $3.146(.353)$ \\
\hline \multicolumn{4}{|l|}{ Manifest Intercepts: } \\
\hline$\tau_{m r}$ & $12.005(.819)$ & $12.834(.781)$ & $12.899(.776)$ \\
\hline$\tau_{b d}$ & $3.218(2.064)^{n s}$ & $5.563(2.042)$ & $6.018(2.053)$ \\
\hline$\tau_{c f}$ & $11.032(.952)$ & $12.104(.912)$ & $12.194(.911)$ \\
\hline$\tau_{a s}$ & $16.806(.716)$ & $17.658(.675)$ & $17.839(.690)$ \\
\hline Factor Variances: & & & \\
\hline$\sigma_{0}^{2}$ (initial level) & $15.405(4.556)$ & $14.538(4.320)$ & $14.417(4.275)$ \\
\hline
\end{tabular}


Table 1 - continued from previous page

\begin{tabular}{|c|c|c|c|}
\hline Parameter & 2LCS & $\begin{array}{l}\text { 2LCS with } \\
\text { indicators } \sim \gamma\end{array}$ & $\begin{array}{l}\text { 2LCS with } \\
\text { indicators } \sim \gamma_{i}\end{array}$ \\
\hline$\sigma_{s}^{2}$ (additive slope) & $.165(.191)^{n s}$ & $.130(.175)^{n s}$ & $.104(.165)^{n s}$ \\
\hline$\sigma_{0 s}\left(\operatorname{Cov}\left(l y_{0}, l y_{s}\right)\right)$ & $1.992(.707)$ & $1.884(.592)$ & $1.831(.572)$ \\
\hline $\begin{array}{l}\sigma_{\Delta f r}^{2} \text { (dynamic vari- } \\
\text { ance) }\end{array}$ & $1.819(.838)^{*}$ & $1.167(.738)^{n s}$ & $1.118(.713)^{n s}$ \\
\hline Residual Variances: & & & \\
\hline$\sigma_{m r}^{2}$ & $15.534(1.454)$ & $15.630(1.465)$ & $15.699(1.465)$ \\
\hline$\sigma_{b d}^{2}$ & 105.604 (10.688) & $101.953(10.704)$ & $102.539(10.734)$ \\
\hline$\sigma_{c f}^{2}$ & $19.255(2.009)$ & $19.382(2.013)$ & $19.330(2.002)$ \\
\hline$\sigma_{a s}^{2}$ & $8.029(.862)$ & $8.604(.912)$ & $8.363(.894)$ \\
\hline$\sigma_{m r_{t}, m r_{t+1}}$ & $2.936(2.748)^{n s}$ & $4.0924(2.520)^{n s}$ & $4.335(2.473)^{n s}$ \\
\hline$\sigma_{b d_{t}, b d_{t+1}}$ & $54.877(9.496)$ & $49.774(9.693)$ & $49.989(9.715)$ \\
\hline$\sigma_{c f_{t}, c f_{t+1}}$ & $.586(2.972)^{n s}$ & $.451(2.890)^{n s}$ & $.380(2.873)^{n s}$ \\
\hline$\sigma_{a s_{t}, a s_{t+1}}$ & $2.261(1.286)^{n s}$ & $3.104(1.292)^{*}$ & $2.833(1.295)^{*}$ \\
\hline Model Fit: & & & \\
\hline $\log (L)$ & -4362.666 & 34629.720 & 34633.203 \\
\hline degrees of freedom & 889 & 4133 & 4130 \\
\hline no. of parameters & 21 & 22 & 25 \\
\hline AIC & 8767.33 & -69215.44 & -69216.41 \\
\hline $\mathrm{BIC}$ & 8836.07 & -69143.43 & -69134.58 \\
\hline
\end{tabular}

$\mathbb{1}^{*}$ : the estimates were significant at .05 level but not .01

${ }^{n s}:$ not significant at .05 level

All the other estimates were significant at $p<.01$ level of significance 


\section{Table 2}

Parameters of Latent Change Score Model (LCS) with and without the retest covariate

\begin{tabular}{|c|c|c|c|}
\hline Parameter & LCS without Retest & $\mathrm{LCS} \sim \gamma$ & $\mathrm{LCS} \sim \gamma_{i}$ \\
\hline \multicolumn{4}{|l|}{ Regressions: } \\
\hline$\beta$ (autoproportion) & $-.193(.020)$ & $-.201(.024)$ & $-.203(.033)$ \\
\hline$\gamma$ (retest coefficient) & - & $.199(.032)$ & $\gamma_{1}=.184(.058)$ \\
\hline & & & $\gamma_{2}=.227(.047)$ \\
\hline & & & $\gamma_{3}=.175(.066)$ \\
\hline \multicolumn{4}{|l|}{ Factor Means: } \\
\hline$\mu_{0}$ & $-1.794(.094)$ & $-1.637(.104)$ & $-1.637(.114)$ \\
\hline$\mu_{s}$ & $.209(.012)$ & $.154(.015)$ & $.153(.016)$ \\
\hline \multicolumn{4}{|l|}{ Factor Variances: } \\
\hline$\sigma_{0}^{2}($ initial level $)$ & $.330(.093)$ & $.377(.100)$ & $.382(.102)$ \\
\hline$\sigma_{s}^{2}$ (additive slope) & $.009(.003)$ & $.008(.004)$ & $.008^{n s}(.004)$ \\
\hline$\sigma_{0 s}\left(\operatorname{Cov}\left(l y_{0}, l y_{s}\right)\right.$ & $.040(.014)$ & $.038(.013)$ & $.037(.013)$ \\
\hline$\sigma_{e}^{2}($ residual $)$ & $.106(.013)$ & $.094(.012)$ & $.094(.012)$ \\
\hline \multicolumn{4}{|l|}{ Model Fit: } \\
\hline$\chi^{2}($ chi square $)$ & 138.94 & 306.24 & 305.47 \\
\hline degrees of freedom & 128 & 337 & 335 \\
\hline no. of parameters & 7 & 8 & 10 \\
\hline $\mathrm{AIC}$ & 576.65 & 542.68 & 545.91 \\
\hline $\mathrm{BIC}$ & 599.56 & 569.11 & 578.94 \\
\hline RMSEA & .021 & .000 & .000 \\
\hline$(90 \% \mathrm{CI})$ & $(.000, .042)$ & $(.000, .013)$ & $(.000, .014)$ \\
\hline
\end{tabular}

$n s$ : Estimate was not significant at .05 level

All the other estimates were significant at $p<.05$ level 


\section{Table 3}

Parameters of LCS with practice latent covariate

\begin{tabular}{|c|c|}
\hline Parameter & LCS with Practice latent variable \\
\hline Regressions: & \\
$\beta$ (auto-proportion) & -.201 \\
Factor Means: & \\
$\mu_{0}$ & -1.637 \\
$\mu_{s}$ & .154 \\
$\mu_{p}$ & .199 \\
Factor Variances: & .378 \\
$\sigma_{0}^{2}$ (initial level) & .008 \\
$\sigma_{s}^{2}$ (additive slope) & $2.3 e-08^{n s}$ \\
$\sigma_{p}^{2}$ (practice) & .037 \\
$\sigma_{0 s}$ (Cov $\left(l y_{0}, l y_{s}\right)$ & .094 \\
$\sigma_{e}^{2}$ (residual) & \\
Model Fit: & 526.69 \\
$-2 \log (\mathrm{L})$ & 544.69 \\
AIC & 598.79 \\
BIC & \\
\hline
\end{tabular}

${ }^{n s}$ : Estimate was not significant at .05 level

All other estimates were significant at $p<.01$ level 


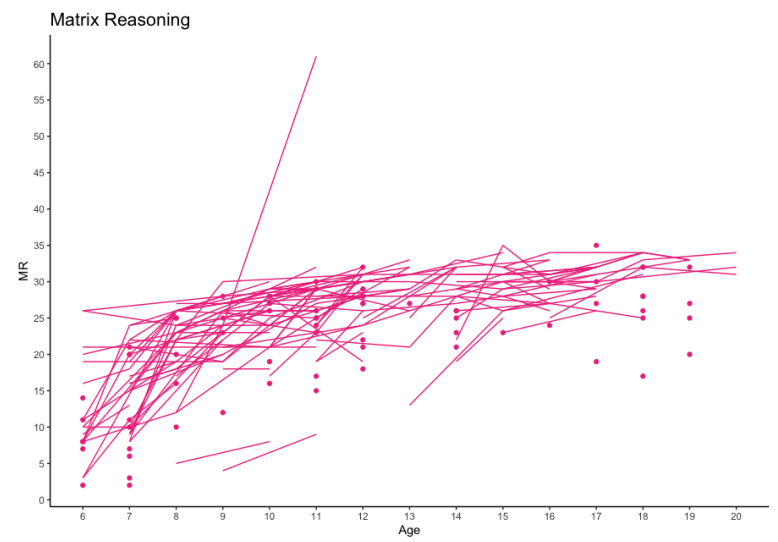

(a) Matrix Reasoning

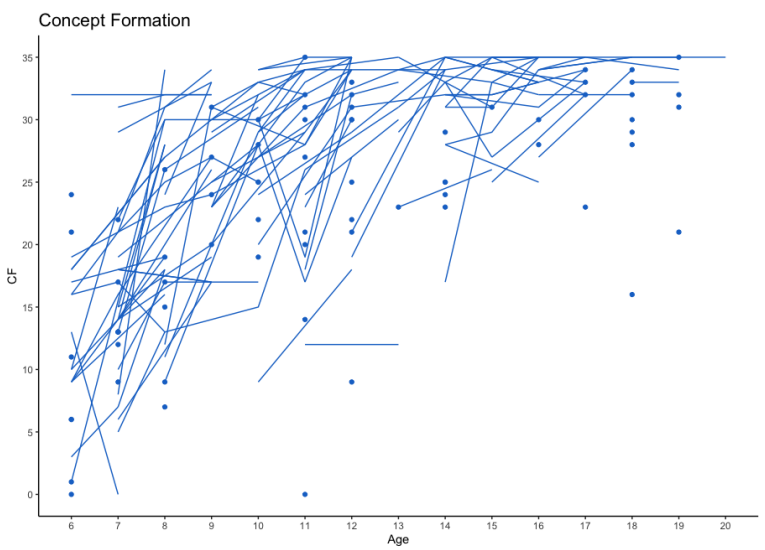

(c) Concept Formation

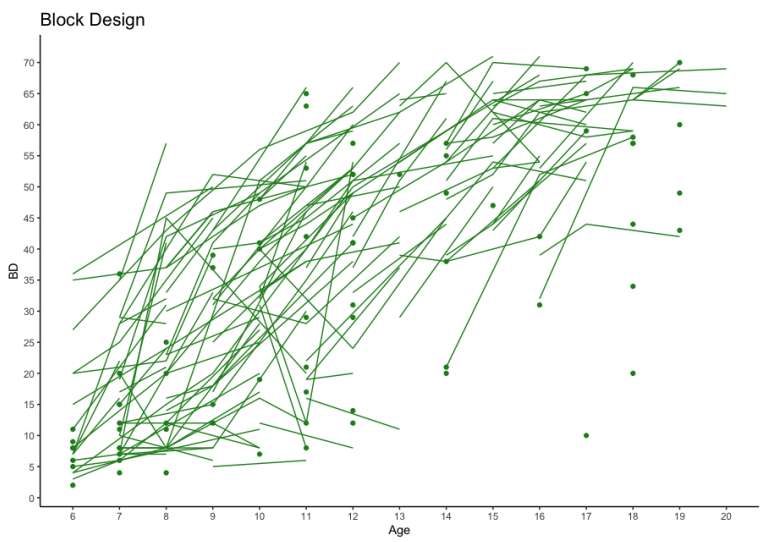

(b) Block Design

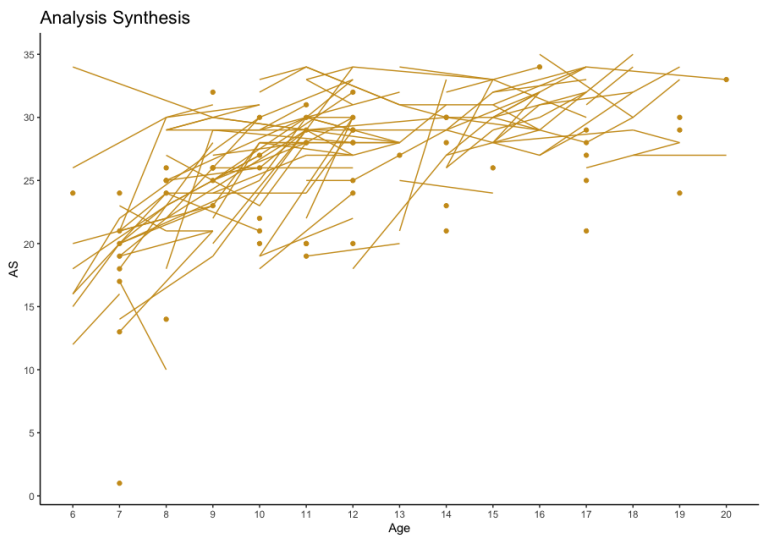

(d) Analysis Synthesis

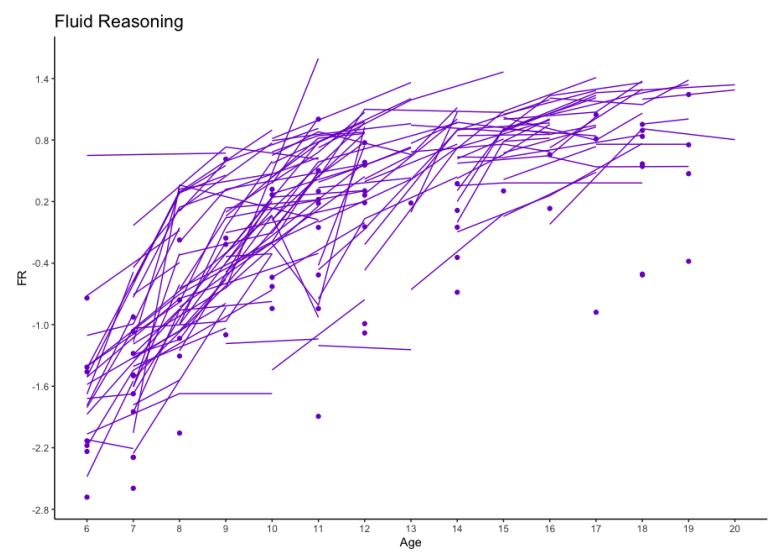

\section{(e) Fluid Reasoning Measure}

\section{Figure 1}

Plots of the observed data for each measure of fluid reasoning. Here, a point is for individuals with only one observation and individuals with multiple data are joined by a line. 


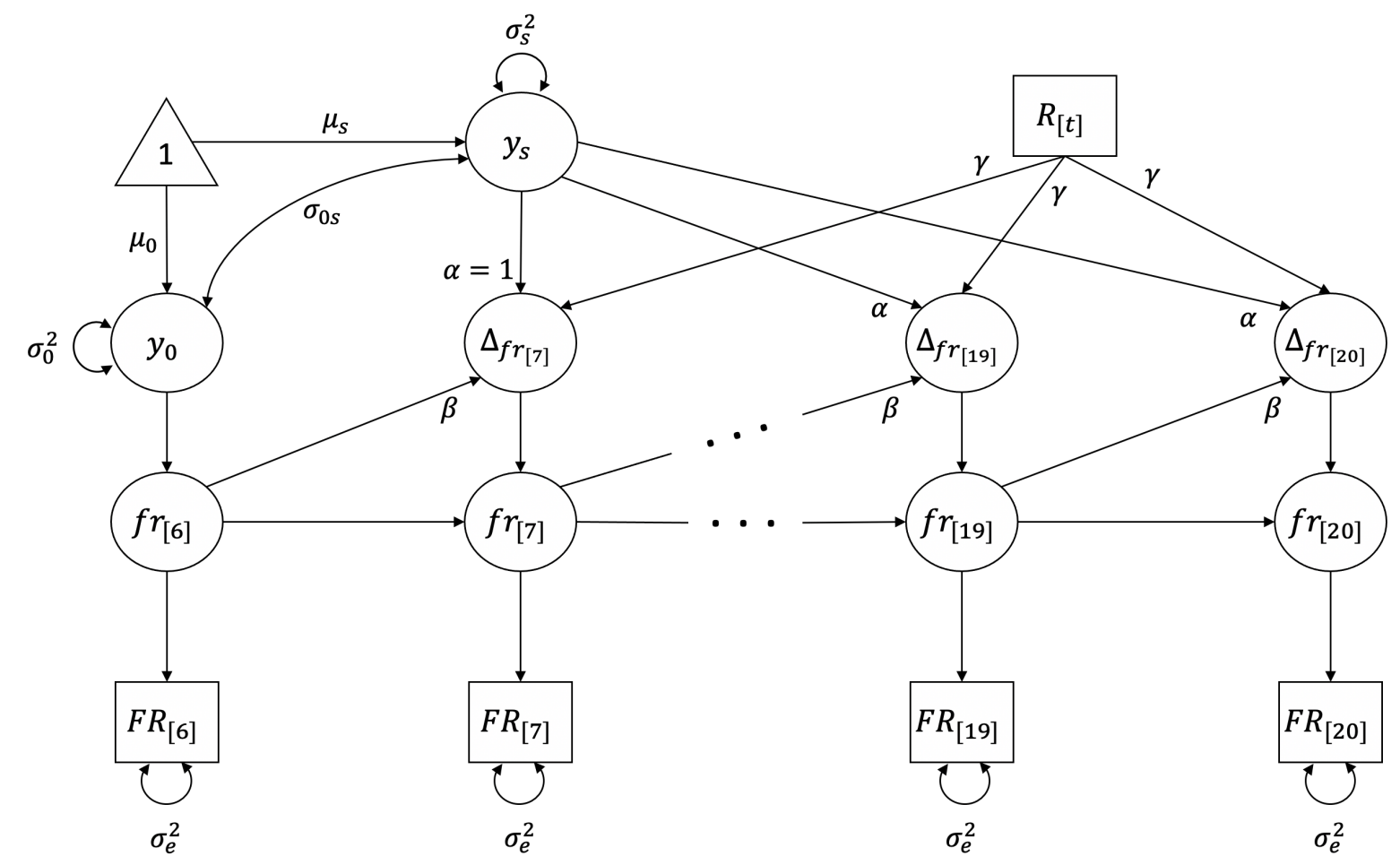

Figure 2

Latent Change Score model with retest effects on the latent changes 


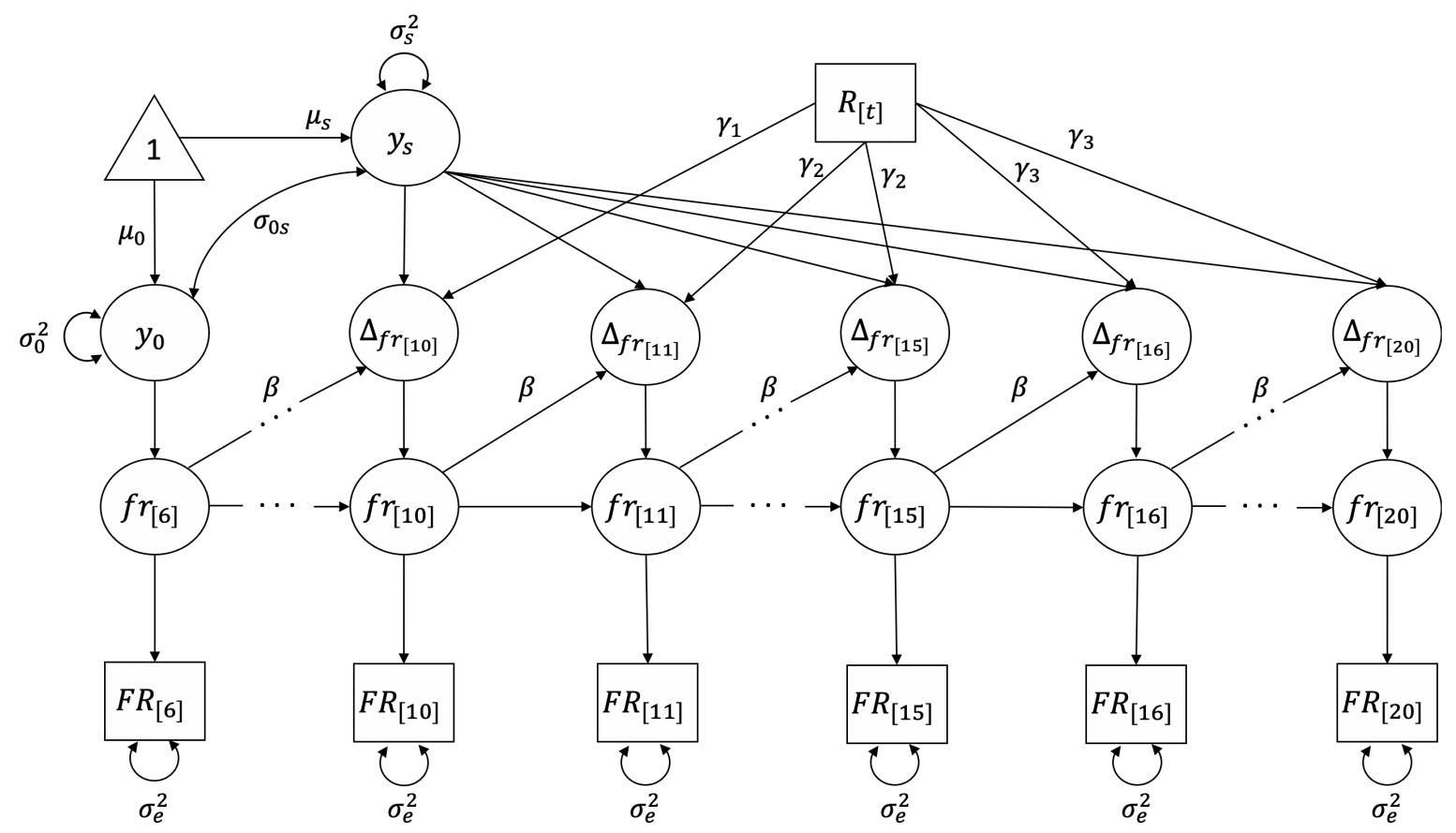

Figure 3

Latent Change Score model with retest effects differences across age groups 


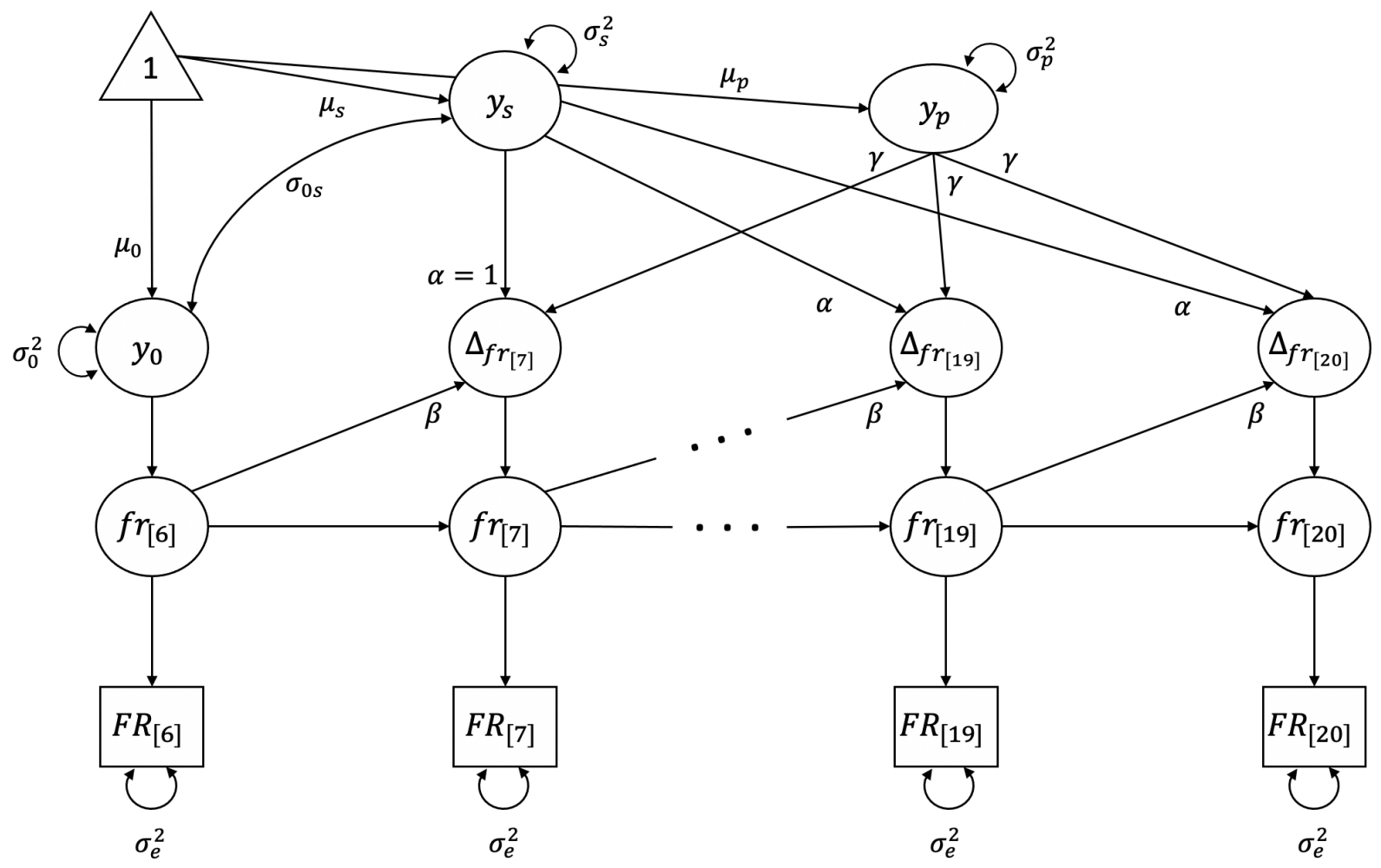

Figure 4

Latent Change Score model with retest as a latent variable loading on the latent changes 


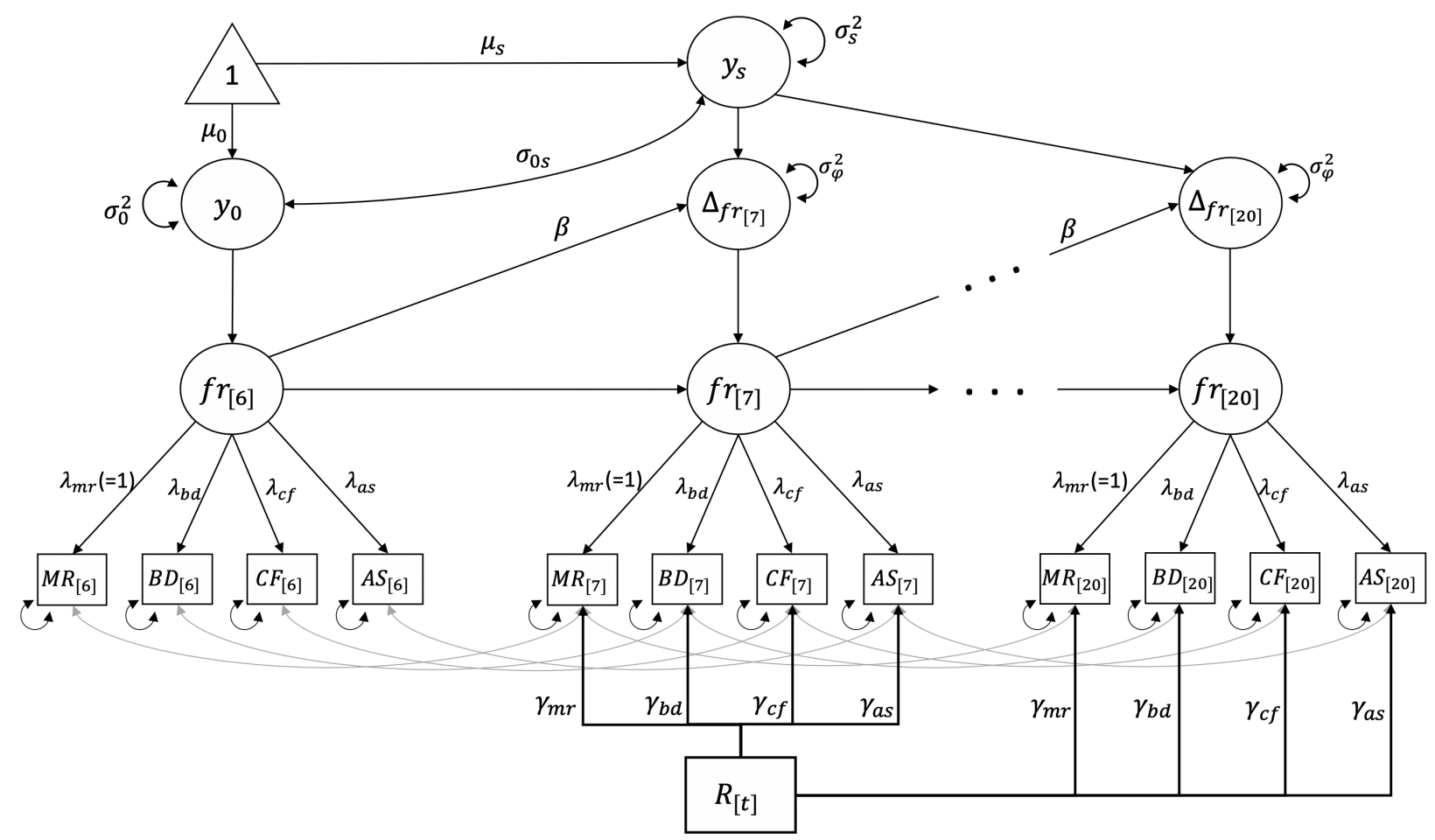

Figure 5

2nd-Order Latent Change Score model with retest as a covariate on the manifest variables 


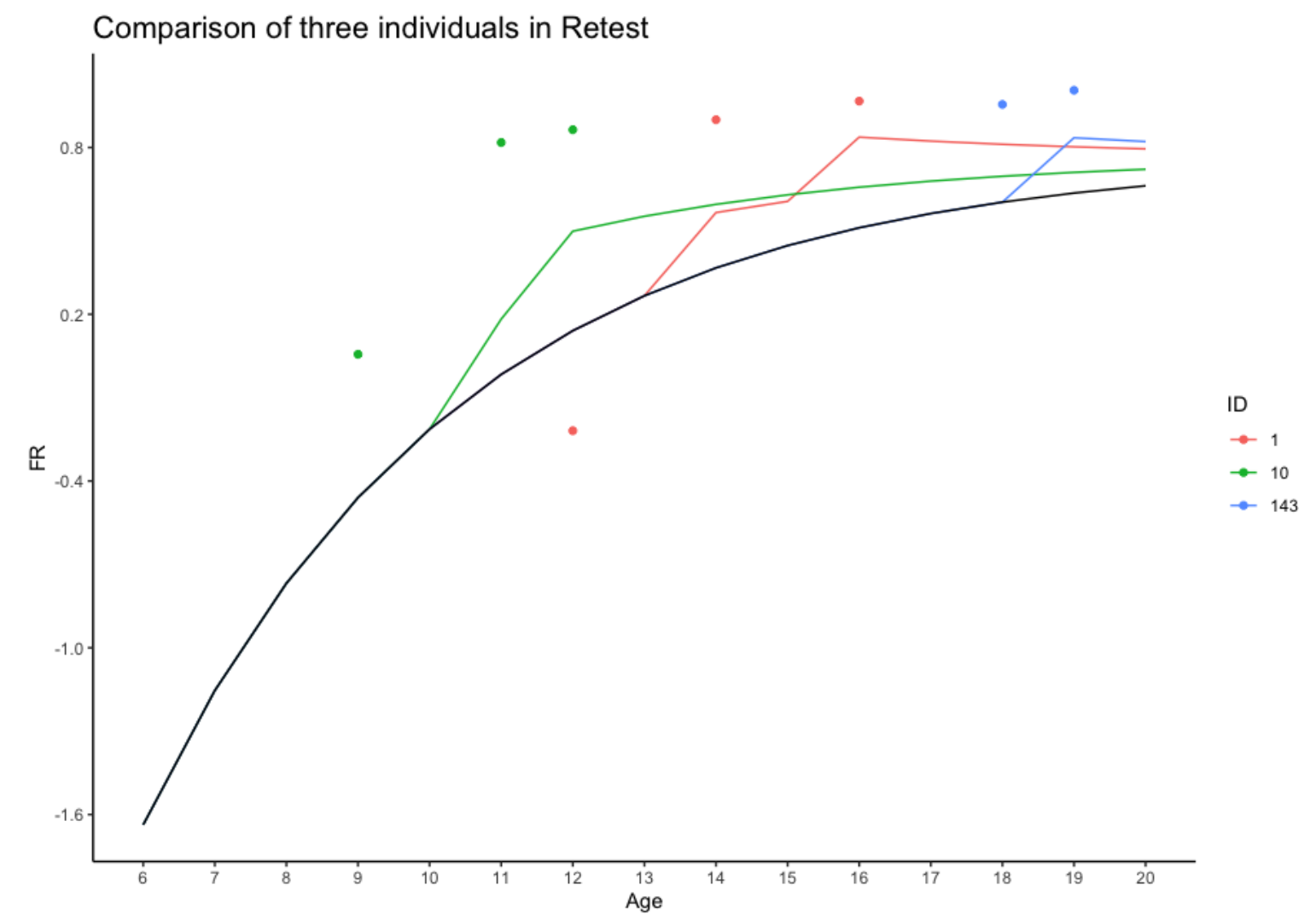

\section{Figure 6}

Comparison of Retest Gain in Univariate LCS across three individuals. Here, the black line indicates the expectations trajectory of Fluid Reasoning true score variables without any retest. We also show three individuals and their observed scores given by dots and the expected trajectory accounting for retest by lines. 


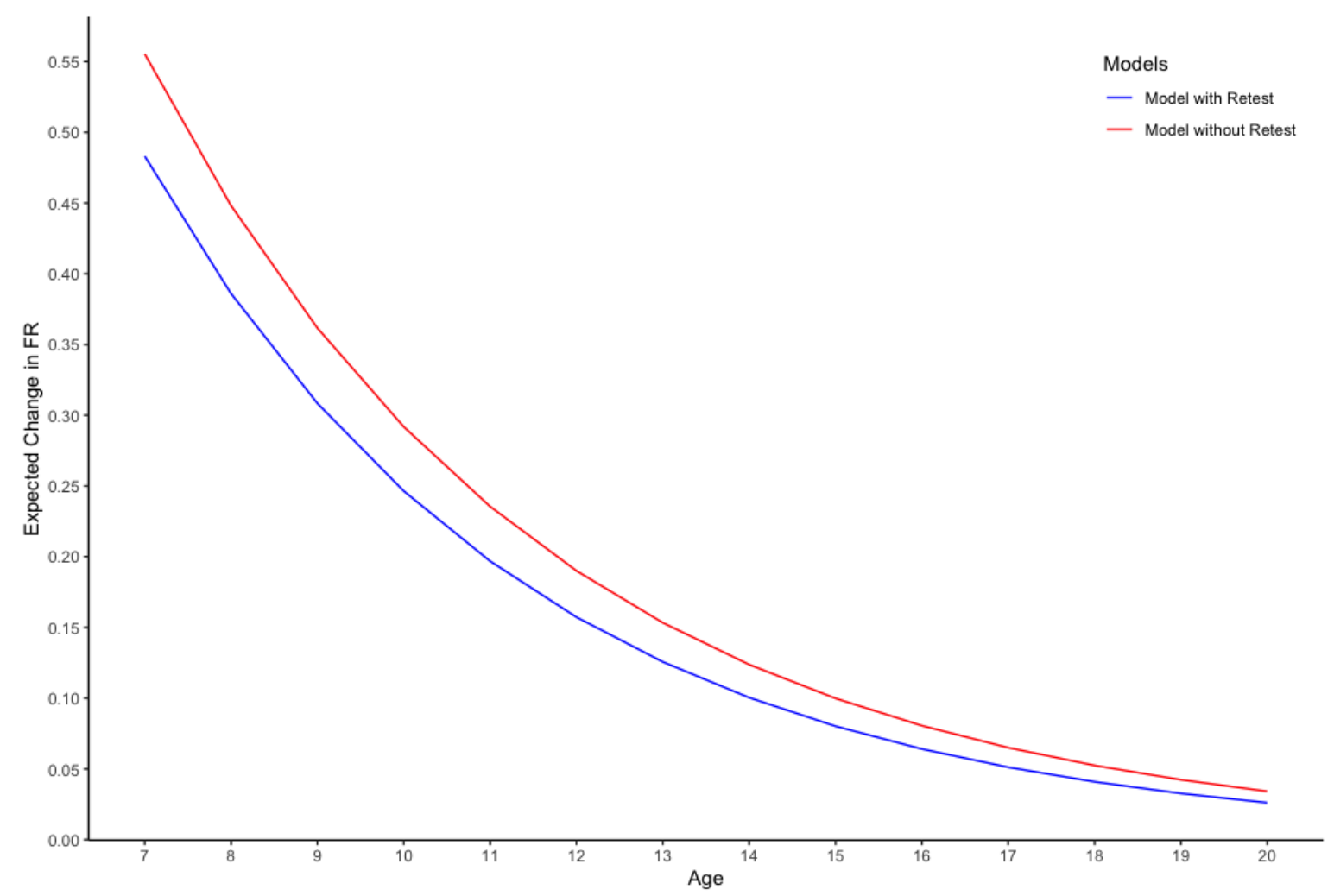

Figure 7

Comparison of trajectory of expected changes in model with retest and without retest in a univariate LCS 


\section{Appendix A}

\section{Table}

\section{Table A1}

Parameters of Multivariate LCS with/without retest covariate (including residual covariances)

\begin{tabular}{|c|c|c|c|}
\hline Parameter & 2LCS & $\begin{array}{l}\text { 2LCS with } \\
\text { indicators } \sim \gamma\end{array}$ & $\begin{array}{l}\text { 2LCS with } \\
\text { indicators } \sim \gamma_{i}\end{array}$ \\
\hline \multicolumn{4}{|l|}{ Loadings: } \\
\hline$\lambda_{m r}$ & $1^{+}$ & $1^{+}$ & $1^{+}$ \\
\hline$\lambda_{b d}$ & $2.825(0.137)$ & $2.934(0.147)$ & $2.961(0.157)$ \\
\hline$\lambda_{c f}$ & $1.210(0.061)$ & $1.220(0.064)$ & $1.224(0.067)$ \\
\hline$\lambda_{a s}$ & $0.745(0.041)$ & $0.726(0.043)$ & $0.758(0.046)$ \\
\hline Regressions: & & & \\
\hline$\beta$ (autoproportion) & $-0.183(0.021)$ & $-0.178(0.022)$ & $-0.176(0.022)$ \\
\hline$\gamma$ (retest coefficient) & & $1.577(0.288)$ & $\begin{array}{l}\gamma_{m r}=2.194(0.458) \\
\gamma_{b d}=2.253(1.180)^{n s} \\
\gamma_{c f}=2.284(0.558) \\
\gamma_{a s}=0.885(0.378)^{*}\end{array}$ \\
\hline \multicolumn{4}{|l|}{ Factor Means: } \\
\hline$\mu_{0}$ & $0^{+}$ & $0^{+}$ & $0^{+}$ \\
\hline$\mu_{s}$ & $3.694(0.366)$ & $3.201(0.351)$ & $3.089(0.345)$ \\
\hline \multicolumn{4}{|l|}{ Manifest Intercepts: } \\
\hline$\tau_{m r}$ & $12.202(0.794)$ & $12.971(0.763)$ & $13.013(0.759)$ \\
\hline$\tau_{b d}$ & $3.032(1.983)^{n s}$ & $5.425(1.997)$ & $5.826(2.003)$ \\
\hline$\tau_{c f}$ & $11.332(0.939)$ & $12.348(0.907)$ & $12.417(0.906)$ \\
\hline$\tau_{a s}$ & $17.031(0.700)$ & $17.750(0.655)$ & $17.978(0.673)$ \\
\hline Factor Variances: & & & \\
\hline
\end{tabular}


Table A1 - continued from previous page

\begin{tabular}{|l|l|l|l|}
\hline Parameter & 2LCS & $\begin{array}{l}\text { 2LCS with } \\
\text { indicators } \sim \gamma\end{array}$ & $\begin{array}{l}\text { 2LCS with } \\
\text { indicators } \sim \gamma_{i}\end{array}$ \\
\hline$\sigma_{0}^{2}$ (initial level) & $14.832(4.340)$ & $14.381(4.196)$ & $14.387(4.183)$ \\
$\sigma_{s}^{2}($ additive slope) & $0.321(0.184)^{n s}$ & $0.287(0.187)^{n s}$ & $0.264(0.182)^{n s}$ \\
$\sigma_{0 s}\left(\operatorname{Cov}\left(l y_{0}, l y_{s}\right)\right)$ & $1.957(0.615)$ & $1.830(0.533)$ & $1.786(0.516)$ \\
$\sigma_{\Delta f r}^{2}($ dynamic vari- & $0.638(0.688)^{n s}$ & $0.186(0.610)^{n s}$ & $0.139(0.592)^{n s}$ \\
ance $)$ & & & \\
Residual Variances: & $15.756(1.423)$ & $15.622(1.395)$ & $15.646(1.389)$ \\
$\sigma_{m r}^{2}$ & $101.065(10.414)$ & $97.990(10.284)$ & $98.749(10.311)$ \\
$\sigma_{b d}^{2}$ & $20.802(2.038)$ & $20.824(2.009)$ & $20.797(2.000)$ \\
$\sigma_{c f}^{2}$ & $8.357(0.856)$ & $8.810(0.884)$ & $8.596(0.866)$ \\
$\sigma_{a s}^{2}$ & & & \\
Model Fit: & 4377.71 & 34614.73 & 34618.47 \\
Log(L) & 792 & 4137 & 4134 \\
degrees of freedom & 17 & -69193.46 & -69194.94 \\
no. of parameters & 8789.41 & -69134.55 & -69126.21 \\
AIC & 8845.05 &
\end{tabular}

$2^{*}:$ the estimates were significant at .05 level but not .01 ${ }^{n s}$ : not significant at .05 level

All the other estimates were significant at $p<.01$ level of significance 


\section{Appendix B}

\section{Figure}

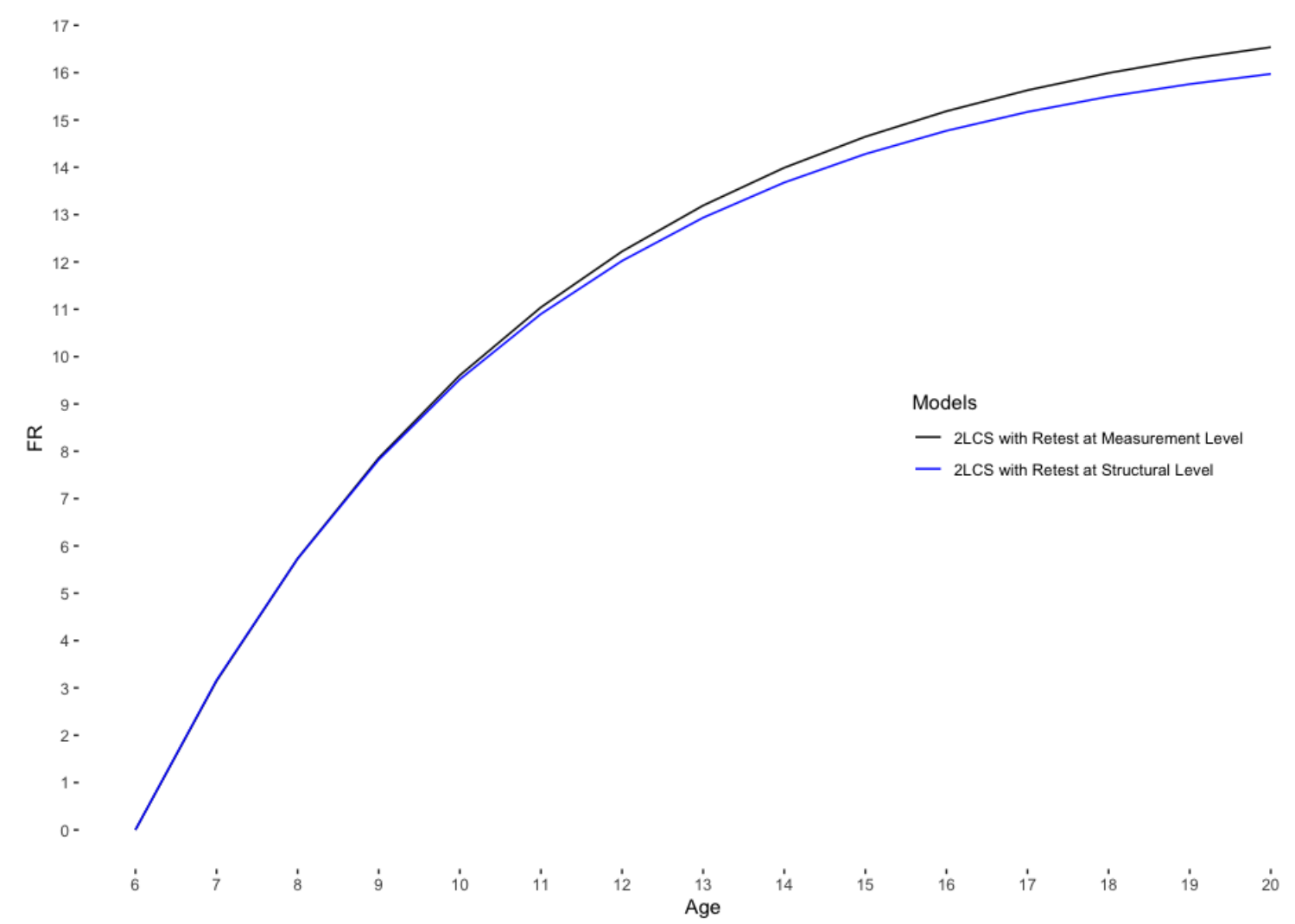

\section{Figure B1}

Comparison of Fluid Reasoning for 2LCS without any individualised retest gains. We used Retest covariate from the univariate framework to fit Retest at the structural level since there was no one retest in a multivariate framework. 